J. Appl. Numer. Optim. 1 (2019), No. 2, pp. 103-105

Available online at http://jano.biemdas.com

https://doi.org/10.23952/jano.1.2019.2.01

\title{
EDITORIAL A SPECIAL ISSUE DEDICATED TO BORIS POLYAK
}

This special issue aims to honor Professor Boris Polyak's outstanding scientific contributions to the field of optimization by collecting some of the most recent advancements in optimization and its applications. During the last six decades, Professor Boris Polyak made fundamental contributions to various aspects of deterministic and stochastic optimization. Professor Boris Polyak is an original and brilliant mathematician, who put forth numerous novel ideas and concepts that strengthen all branches of optimization. His well-known book on optimization has educated generations of researchers, and his heavily cited papers provide a wealth of innovative ideas.

This special issue is comprised of seven articles of high scientific merits whose contribution we summarise in the following:

A. Agrawal, S. Barratt, S. Boyd, E. Busseti, and W. M. Moursi, in the paper entitled "Differentiating Through a Cone Program," consider the problem of efficiently computing the derivative of the solution map of a convex cone program. They implicitly differentiate the residual map for the homogeneous self-dual embedding and solve the linear systems of equations by employing an iterative method. This permits the authors to compute the derivative operator and its adjoint effectively. An open-source Python implementation of the new method solves a cone program and returns the derivative and its adjoint as abstract linear maps.

The objective of the article entitled "A Henig conical regularization approach for circumventing the Slater conundrum in linearly $\ell_{+}^{p}$-constrained least-squares problems" by L. Huerga, A. A. Khan and M. Sama is to explore a linearly $\ell_{+}^{p}$-constrained least-squares problem. The authors develop a Henig conical regularization approach as a unified framework to deal with the lack of Slater-type constraint qualification. They provide some stability estimates for the regularized problems. For the separable case, $p \in[1, \infty)$, they give an explicit characterization of the Henig dilating cones associated with $\ell_{+}^{p}$ and the associated regularized KKT systems. For the non-separable case $\ell_{+}^{\infty}$, they provide a condition that ensures that the solution of the least-squares problem can be approximated by regularized solutions whose dual solutions do not contain any finitely additive singular part.

In the paper entitled "Computing minimal elements of finite families of sets w.r.t. preorder relations in set optimization," C. Günther, E. Köbis, and N. Popovici, propose new algorithms for computing all minimal elements of a nonempty finite family of sets in a real linear space, with respect to a preorder relation defined on the power set of that space. One of the new algorithms consists of two subsequent 
(forward-backward) reduction procedures, akin to the classical Jahn-Graef-Younes method. Another algorithm involves a pre-sorting procedure with respect to a strongly increasing real-valued function, followed by a single (forward) reduction procedure. Numerical experiments in MATLAB allow the authors to compare the algorithms for special test families of line segments concerning $\ell$-type, $u$-type and $s$-type preorder relations, currently used in set optimization.

A. Gibali, N.H. Ha, N.T. Thuong, T.H. Trang, and N.T. Vinh in the paper entitled "Polyak's gradient method for solving the split convex feasibility problem and its application," are concerned with finding minimum-norm solutions of the split convex feasibility problem in real Hilbert spaces. The authors study and analyze the convergence of Byrne's well-known C.Q. algorithm, which is Polyak's gradient method applied to an equivalent minimization model of the split convex feasibility problem. A new self-adaptive C.Q. variant is proposed.

The article entitled "Three convergence results for inexact orbits of nonexpansive mappings" by A. J. Zaslavski presents the convergence of inexact iterates of nonexpansive mappings which take a nonempty, closed subset of a complete metric space into the space, under the presence of summable errors. The new results generalize the results known in the literature for nonexpansive self-mappings of the complete metric space.

V. Babenko and V. Babenko, in the paper entitled "Best Approximation, Optimal Recovery, and Landau Inequalities for Derivatives of Hukuhara-type in function $L$-spaces," consider the problem of approximation of unbounded positively homogeneous operators in $L$-spaces by using Lipschitz operators. The authors study its connection to the problem of computing modulus of continuity of the unbounded operator on the class of elements and the problem of optimal recovery of an unbounded operator by a Lipschitz one on the class of elements given with an error. Furthermore, in $L$-spaces and for positively homogeneous operators, the connection of the above-stated problems with inequalities of Landau-Kolmogorov type is given. Some interesting applications of the main results are given.

The objective of G. Pany, O. Chadli, and R.N. Mohapatra, in the paper entitled "Generalized monotone mixed equilibrium problems in FC-Spaces: Existence and approximation,"is to study a class of mixed equilibrium problems under extended generalized $\alpha-\eta$ monotonicity in finitely continuous topological spaces. Existence of the solution to the problem is shown by relaxing the convexity structure and the linearity condition using relatively Knaster-Kuratowski-Mazurkiewicz principle. The authors propose an iterative scheme using the auxiliary principle technique and provide the convergence analysis.

Finally, we give our sincerest gratitude to all the authors who have contributed to this special issue and to the reviewers.

Akhtar A. Khan

School of Mathematical Sciences

Rochester Institute of Technology, Rochester, USA

E-mail address: aaksma@rit.edu 
Departamento de Matemática Aplicada Universidad Nacional de Educación a Distancia, Madrid, Spain E-mail address: msama@ind.uned.es

Alexandra B. Smirnova Department of Mathematics and Statistics Georgia State University, Atlanta, USA E-mail address: asmirnova@gsu.edu 